\title{
Clinical study of primary progressive multiple sclerosis in Northern Ireland, UK
}

\author{
G V McDonnell, S A Hawkins
}

\begin{abstract}
Objective-To investigate the clinical and demographic characteristics of primary progressive multiple sclerosis (PPMS) in Northern Ireland and to establish a database of such patients for genetic and immunological studies and future therapeutic trials.

Methods-Diagnosis and categorisation were performed by two neurologists, potential cases being identified from the following sources: neurology outpatient clinics; neurology inpatients; a review of hospital discharges; and an ongoing epidemiological study of multiple sclerosis in Northern Ireland. Only those with a progressive course from onset and a clear history of no prior relapses were accepted. Potential cases were invited for interview and assessment, the minimal record of disability (MRD) being established.

Results-One hundred and eleven cases of PPMS have been identified, 63 women and 48 men (ratio 1.3:1), with a mean age at onset of 39.5 (SD 11.0) (range 17-66)years, and mean disease duration of 13.6 (SD 9.3)years. The mean interval between onset and diagnosis was 4.7 (SD 4.2) years. Nineteen patients $(17.1 \%)$ did not satisfy the requirements for any category in the Poser criteria. Motor disturbance was the commonest mode of onset $(67.6 \%)$ with visual loss occurring only rarely at onset (3.6\%). Kurtzke EDSS scores were concentrated at the upper end of the scale with a median of 6.0 and levels of unemployment and financial dependence were high.
\end{abstract}

Conclusions-PPMS in Northern Ireland has a generally later age at onset, lower female preponderance, and predominantly motor onset compared with other subgroups of multiple sclerosis. The delay to diagnosis reflects the often insidious onset and the nature of the clinical course makes application of the Poser criteria difficult. Levels of neurological impairment, disability, and handicap as measured by the MRD are high.

(F Neurol Neurosurg Psychiatry 1998;64:451-454)

Keywords: multiple sclerosis; primary progressive multiple sclerosis; Northern Ireland

Quin House, Royal Victoria

Hospital, Grosvenor Road,

Belfast BT12 6BA, Northern

Ireland, UK. Telephone 004

1232240503 ext 4325; fax

00441232235258 .

Received 15 August 1997 and in revised form 10 October 1997

Accepted 16 October 1997

There is mounting evidence to suggest that patients with a primary progressive course of multiple sclerosis (PPMS) exhibit a distinct profile in the range of multiple sclerosis and may even represent a separate disease entity.
The clinical course is typically devoid of the relapses and remissions characteristic of most patients with multiple sclerosis. PPMS is estimated to represent around $10 \%-20 \%{ }^{1}$ of all cases although because of differing definitions and methods of patient selection, estimates vary significantly from $9 \%^{2}$ to $26 \%^{3}$ in clinic based surveys and from $10 \%^{4}$ to $37.4 \%{ }^{5}$ in population studies, a recent epidemiological study in Northern Ireland indicating the proportion of patients with PPMS to be $12.5 \% .^{6}$

Patients with PPMS have been found to have a very different demographic profile to those with the relapsing-remitting (RRMS) or secondary progressive (SPMS) forms of the disease, often shown by an older age of onset and a lesser female predominance. The commonest mode of presentation has characteristically been seen to be with motor disturbance rather than with the visual and sensory symptoms which are the most frequent initial disturbances in RRMS. The evidence for heterogeneity in multiple sclerosis does, however, extend beyond the demographic and the clinical. Brain MRI has shown that whereas patients with clinically benign multiple sclerosis or SPMS often have extensive abnormalities, patients with PPMS have remarkably few lesions ${ }^{78}$ and new lesions seen on MRI less often enhance with gadolinium in PPMS than in SPMS, despite similar levels of disability and rates of progression of disease.$^{9}$ The corollary that PPMS may be a less inflammatory form of multiple sclerosis has been supported by a postmortem evaluation. ${ }^{10}$ New MR techniques have suggested that axonal loss plays an important part in the development of the disability seen in PPMS and perhaps explains the apparent discrepancy between disability and the lack of lesions seen on both cranial and spinal cord MRI. ${ }^{11-13}$ Potential immunological differences between PPMS and the other multiple sclerosis subgroups have been found ${ }^{14-16}$ and some studies have also pointed to an immunogenetic heterogeneity with differing HLA associations. ${ }^{17-19}$

In view of this emerging distinctive profile of PPMS, we have sought to investigate the clinical course, demography, degree of impairment, disability, and handicap in a significant population of such patients in Northern Ireland, thereby establishing a database for both genetic and immunological studies and for potential future therapeutic trials.

\section{Methods}

For the purposes of this study we have defined PPMS as multiple sclerosis which is progressive from the onset with no relapses or 


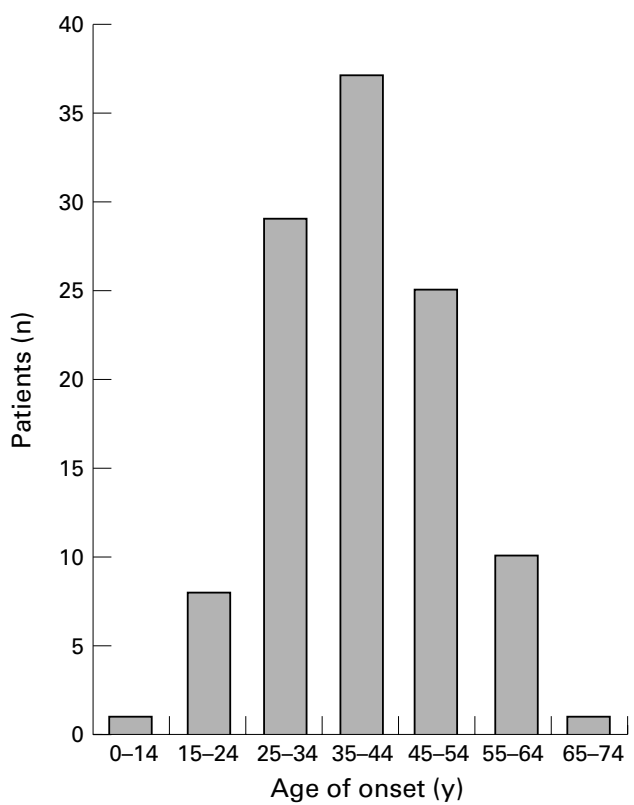

Figure 1 Age of onset of patients with primary progressive multiple sclerosis.

remissions, compatible with a recent consensus definition of this and other subgroups. ${ }^{20}$ Within this definition we have allowed for periods of apparent stability during which little or no progression occurs and we have also accommodated periods of acute worsening associated with other intercurrent illnesses.

The neurological service in Northern Ireland is provided by a Regional Centre with inpatient and diagnostic facilities at the Royal Victoria Hospital in Belfast together with outreach clinics around the country. Potential cases of PPMS were identified by a consultant neurologist from those attending neurology outpatient clinics, neurology inpatients at the Royal Victoria Hospital, a review of hospital discharges, and from a prevalence study of multiple sclerosis undertaken in the Coleraine, Ballymena, Ballymoney, and Moyle districts of Northern Ireland. The neurological and medical records of such potential cases were reviewed and those documented as having a progressive course from onset without relapses or remissions were then invited for interview and assessment.

Assessments took place at a neurology outpatient clinic, in the patients' local health centres, or in their own homes. The assessment involved interview with regard to relevant clinical history including date of onset, date of diagnosis, nature of initial presentation, subsequent clinical course, and family history. Date of onset was obtained from the patient where possible, from the medical records if not, and was defined as the time of the first neurological symptom that could be attributed to the disease. The date of diagnosis was obtained from the medical records. Patients were questioned specifically and closely about the possible occurrence of previous relapses. Where doubt existed or where a transitional course was suspected, patients were excluded. Those with insufficient evidence of dissemination in space and who had not undergone appropriate investigation to exclude a compressive or intrinsic spinal cord lesion (myelography or MRI) were also excluded. The minimal record of disability (MRD) including the expanded disability status scale of Kurtzke (EDSS), ${ }^{21}$ functional system (FS) scores, incapacity status scale, (ISS) ${ }^{22}$ and environmental status scale $(\mathrm{ESS})^{23}$ was determined in each case.

Approval was obtained for the study from the Queen's University of Belfast research ethics committee.

\section{Results}

After exclusion of those with a definite or possible history of relapse, those with a diagnosis other than multiple sclerosis, and those patients in whom a compressive cord lesion had not been satisfactorily excluded, we have identified 111 cases of multiple sclerosis with a primary progressive course.

There were 63 women and 48 men giving a ratio of 1.3:1. The mean age of the group at the time of assessment was 53.1 (SD 12.4) (range 25-75) years. The mean ages for women and men were 54.6 (SD 12.0) and 51.0 (SD 12.7) years respectively. The mean age of onset was 39.5 (SD 11.0) (range 17-66) years. The figures for women and men were 41.6 (SD 11.2) and 36.7 (SD 10.1) respectively. Figure 1 shows the distribution of patients by age of onset. The mean disease duration for the whole group was 13.6 (SD 9.3) years and the mean interval between onset and diagnosis was 4.7 (SD 4.2) years.

Each patient was assigned where possible to one of the diagnostic categories of the Poser criteria $^{24}$ — namely, clinically definite (CDMS), laboratory supported definite (LSDMS), clinically probable (CPMS), or laboratory supported probable (LSPMS). Those not satisfying the criteria for any of these categories were designated as "suspected multiple sclerosis". Due to the lack of relapses in PPMS and therefore the absence of a second genuine "attack", no patients could be classified as having either CDMS or LSPMS. Forty one patients $(36.9 \%)$ were designated as having LSDMS and 51 were considered to have CPMS (45.9\%). Nineteen patients $(17.1 \%)$ were classified as "suspected". Three of these were excluded from the classification due to onset of symptoms after the age of 59 years, two otherwise fulfilling the criteria for LSDMS and one satisfying the criteria for CPMS. Of the remaining 16 , seven had oligoclonal bands in the CSF and one had an abnormal MRI. This left eight patients with a progressive myelopathy. Compressive and vascular spinal cord lesions had been ruled out in all and none had a family history of progressive spastic paraparesis. Of these eight patients, four had CSF analysed for oligoclonal bands with a negative result and the remaining four had not been tested.

The symptoms at onset could be established in all cases. The commonest mode of presentation was with motor disturbance ( 75 patients; $67.6 \%$ ), followed by sensory (20 patients; $18.0 \%$ ), brainstem or cerebellar (12 patients; $10.8 \%$ ), sphincter disturbance (five patients; 


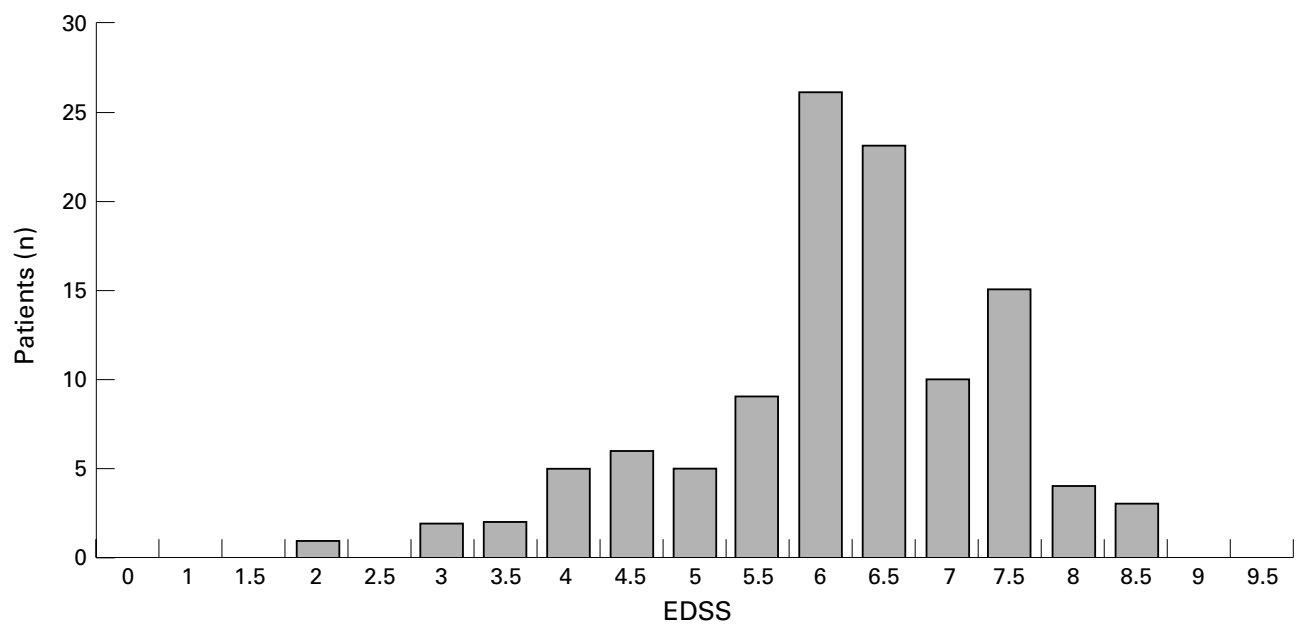

Figure 2 Kurtzke EDSS scores in the primary progressive multiple sclerosis population.

$4.5 \%$ ), and progressive visual loss (four patients; $3.6 \%)$. Five patients $(4.5 \%)$ had some other form of disturbance at onset and 10 $(9.0 \%)$ had more than one of these sets of symptoms at onset.

Oligoclonal band testing in CSF was positive in 62 of the 74 patients $(83.8 \%)$ in whom this had been undertaken. Eighty seven patients had measurement of visual and somatosensory evoked responses, of whom 78 had significant delay in one or both $(89.7 \%)$.

Figure 2 shows the Kurtzke EDSS scores and their distribution, which were again available in all cases. The skewing of this distribution towards the upper end of the scale is reflected in the high proportion of patients with a pyramidal functional system (FS) score $>3$ (51 patients $(45.9 \%)$ having a pronounced paraparesis or hemiparesis (function is difficult), moderate tetraparisis (function is reduced but can be sustained for short periods), or monoplegia).

Twenty five of 109 patients $(22.9 \%)$ had severe bladder dysfunction (a minimum of incontinence at least once a day during the previous week or requiring intermittent catheterisation at least once a day) and 61 of 109 patients $(56.0 \%)$ described significant sexual problems attributable to their multiple sclerosis whereas just eight of 109 (7.3\%) were judged to have moderate cognitive impairment or worse. Of these, four had presented with a myelopathy, three with a brain syndrome, and one with urinary disturbance. Sixteen of 110 patients $(14.5 \%)$ were in employment and $103 / 110(93.6 \%)$ were in receipt of at least some external financial support.

\section{Discussion}

This study of PPMS in Northern Ireland serves to reinforce the distinctive nature of this subgroup in the range of multiple sclerosis. Although a female predominance exists, the ratio at $1.3: 1$ is considerably less than that found in population based studies in Northern Ireland and elsewhere. Similar findings in PPMS have been found by others ${ }^{52}$ although it has not been a universal experience. ${ }^{26}{ }^{27}$ The mean age at onset was relatively advanced at
39.5 years. This is in line with previous data, in which mean age at onset has been reported as 43 by Shepherd, ${ }^{28} 37.3$ by Confavreux et al, ${ }^{29}$ and 37.5 by Minderhoud et $a l^{5}$ whereas the corresponding figures for relapsing-remitting cases were $31,29.2$, and 28.8 years respectively. This older age at onset is also compatible with the results of Noseworthy et $a l^{26}$ who discovered that a primary progressive course is the commonest subgroup in patients whose first manifestations of multiple sclerosis are after the age of 50 and is reflected in the data of Leibowitz et $a l^{30}$ and Cazzullo et $a l^{31}$ who found that $57 \%$ and $49 \%$ respectively of those with onset after age 40 had a primary progressive course. Further, Weinshenker et al have shown that with each passing decade, there is a steadily increasing proportion of patients with progressive disease from onset. ${ }^{32}$

The relatively long mean delay between onset of symptoms and actual diagnosis at almost five years reflects the inherent delays in a patient attending a general practitioner and the further interval between referral and assessment by a neurologist but is also indicative of the often insidious onset and slow progression of many cases.

The difficulty of applying the Poser criteria to PPMS is emphasised by this study. The definition of "attacks" in the criteria and the absence of such repeated episodes in the course of PPMS effectively excludes these patients from two of the Poser categories-CDMS and LSPMS. Most patients can, however, be accomodated within one of the other Poser categories $(82.9 \%$ ) although a few (three in this instance) who might have done so are excluded due to age of onset over age 59, again reflecting the generally later age of onset of the group as a whole.

Over two thirds of our patients presented with a motor disturbance at onset, broadly agreeing with others who have found that a considerable proportion of patients with PPMS have a monosymptomatic myelopathy. Minderhoud et a $\bar{l}$ found that $61.1 \%$ of patients with PPMS had pyramidal signs and symptoms at onset whereas only $19.1 \%$ of a remittent group did so. Similarly in the study of Larsen $e t$ 
$a l,{ }^{27} 79.4 \%$ of patients with PPMS had motor weakness at onset, whereas only $16.5 \%$ of patients with relapsing-remitting or secondary progressive disease presented likewise. Conversely, presentation with optic neuritis tends to be rare in PPMS, ${ }^{52732}$ as in our study, but common in relapsing-remitting multiple sclerosis. Progressive visual loss should not however exclude a diagnosis of PPMS having previously been described as the presenting feature of multiple sclerosis. ${ }^{33}$

The Kurtzke EDSS, because it is not an ordinal scale with similar rates of progression through all points and due to its overreliance on ambulatory ability, tends to produce a bimodal distribution when applied to clinic or population surveys involving the full clinical range of multiple sclerosis. In this study of PPMS alone a more skewed distribution results with two thirds of patients (74/111) having EDSS scores of 6.0-7.5. The progressive paraparesis of many patients which is responsible for this, is shown by the large proportion of patients having a high pyramidal FS score $(45.9 \%)$. This degree of neurological impairment with a median EDSS of 6.0 doubtless contributes to the high levels of unemployment (85.55) and financial dependence $(93.6 \%)$ seen in the group. By contrast, the low level of significant cognitive impairment which is in line with the findings of other groups, ${ }^{34}{ }^{35}$ is likely to be explained by the lower cerebral lesion load in PPMS as has been shown by MRI.

The method of ascertainment used in this study, with direct patient interview, employment of two neurologists, and application of a strict definition of PPMS excluding those with a possible transitional course ${ }^{36}$ has led to the recruitment of a relatively "pure" PPMS population. Certainly the resulting data are distinct from those accruing from recent epidemiological studies across the whole multiple sclerosis population in Northern Ireland, while being broadly in line with the results of others investigating this particular subgroup of patients with multiple sclerosis. The group is one of the largest so described and we trust that it will provide a valuable asset in the investigation of the genetic and immunological basis of multiple sclerosis. Perhaps more importantly, we also wish that with the advent of several new immunotherapeutic strategies in the management of multiple sclerosis, this group of patients may be offered some renewed hope with their inclusion in future therapeutic trials.

Dr McDonnell has been a research fellow at the Royal Victoria Hospital in Belfast and the work has also been supported in part by the charity Action Multiple Sclerosis. We acknowledge the invaluable assistance of the following consultant neurologists in the identification of potential patients for this study-Dr JM Gibson, Dr VH Patterson, Dr JI Morrow, Dr JA Lyttle, and Dr TFG Esmonde.

1 Weinshenker BG. Natural history of multiple sclerosis. Ann Neurol 1994;36:S6-11.

2 Bonduelle M, Albaranes R. Etude statistique de 145 cas de la sclerose en plaques. Semaine des Hopitaux de Paris 1962 38:3762-73.

3 Verjans E, Theys P, Delmotte P, et al. Clinical parameters and intrathecal IgG synthesis as prognostic factors in multiple sclerosis. F Neurol 1983;229:155-65.
4 Panelius M. Studies on epidemiological, clinical and Panelius M. Studies on epidemiological, clinical and 1969;45(suppl 39):1-82.

5 Minderhoud JM, van der Hoeven JH, Prange AJA. Course and prognosis of chronic progressive multiple sclerosis. Acta Neurol Scand 1988;78:10-5.

6 McDonnell GV, Hawkins SA. An epidemiological study of multiple sclerosis in Northern Ireland. Neurology 1998 (in press).

7 Thompson AJ, Kermode AG, MacManus DG, et al. Pathogenesis of progressive multiple sclerosis. Lancet 1989;333: 1322-3.

8 Thompson AJ, Kermode AG, MacManus DG, et al. Patterns of disease activity in multiple sclerosis: clinical and magnetic resonance imaging study. BMF 1990;300:631-4.

Thompson AJ, Kermode AG, Wicks D, et al. Major differences in the dynamics of primary and secondary p

10 Revesz T, Kidd D, Thompson AJ, et al. A comparison of the pathology of primary and secondary progressive multiple sclerosis Brain 1994;117:759-65.

11 Losseff NA, Webb SL, O'Riordan JI, et al. Spinal cord atrophy and disability in multiple sclerosis: a new reproducible and sensitive MRI method with potential to monitor disease progression. Brain 1996;119:701-8.

12 Davie CA, Barker GJ, Webb S, et al. A proton spectroscopy study of disability in multiple sclerosis. F Neurol 1996;243: S33.

13 Kidd D, Barker GJ, Tofts PS, et al. The transverse magnetic decay characteristics of long standing lesions and normal Dore-Duffy P, Newman W, Balabanov R, et al Circulating soluble adhesion molecules in cerebrospinal fluid and serum of patients with multiple sclerosis: correlation with disease activity. Ann Neurol 1995;37:55-62.

15 Giovannoni G, Thorpe JW, Kidd D, et al. Soluble E-selectin in multiple sclerosis: clinical and MRI correlation. $f$ Neuroimmunol 1994;54:164.

16 Giovannoni G, Thorpe JW, Kidd D, et al. Soluble E-selectin in multiple sclerosis: raised concentrations in patients with primary progressive disease. I Neurol Neurosurg Psychiatry 1996;60:20-6.

17 Olerup O, Hillert J, Fredrikson S, et al. Primarily chronic progressive and relapsing/remitting multiple sclerosis: two mmunogenetically distinct disease entities. Proc Natl Acad Sci USA 1989;86:7113-7.

18 Hillert J, Gronning M, Nyland $\mathrm{H}$, et al. An immunogenetic heterogeneity in multiple sclerosis. $\mathcal{F}$ Neurol Neurosurg Psychiatry 1992;55:887-90.

19 Weinshenker BG, Santrach P, Schaid D, et al. Association between class II major histocompatibility complex polymorphisms and the course of multiple sclerosis. Neurology 1995;45:A384.

20 Lublin FD, Reingold SC. Defining the clinical course of multiple sclerosis: results of an international survey. Neurology 1996;46:907-11.

21 Kurtzke JF. Rating neurologic impairment in multiple sclerosis: an expanded disability status scale (EDSS). Neurology 1983;33:1444-52.

22 Kurtzke JF. A proposal for a uniform minimal record of disability in multiple sclerosis. Acta Neurol Scand 1981; 64(suppl 87):110-29.

23 Mellerup E, Fog T, Raun N, et al. The socio-economic scale. Acta Neurol Scand 1981;64(suppl 87):130-8

24 Poser CM, Paty DW, Scheinberg L, et al. New diagnostic criteria for multiple sclerosis: guidelines for research protocols. Ann Neurol 1983; 13:227-31.

25 Van Lambalgen RV, Sanders EACM, D'Amaro J. Sex distribution, age of onset and HLA profiles in two types of multiple sclerosis: a role for sex hormones and microbial infections in the development of autoimmunity. $f$ Neurol Sci tions in the devel 76 : $13-21$.

26 Noseworthy J, Paty D, Wonnacott T, et al. Multiple sclerosis after age 50. Neurology 1983;33:1537-44.

27 Larsen JP, Kvaale G, Riise T, et al. Multiple sclerosis-more than one disease? Acta Neurol Scand 1985;72:145-50.

28 Shepherd DI. Clinical features of multiple sclerosis in north-east Scotland. Acta Neurol Scand 1979;60:218-30.

29 Confavreux C, Aimard G, Devic M. Course and prognosis of multiple sclerosis assessed by the computerised data processing of 349 patients. Brain 1980;103:281-300.

30 Leibowitz U, Alter M, Halpern L. Clinical studies of multiple sclerosis in Israel 3: clinical course and prognosis related to age. Neurology 1964;14:926-32.

31 Cazzullo CL, Ghezzi A, Marforio S, et al. Clinical picture of multiple sclerosis with late onset. Acta Neurol Scand 1978; 58:190-6.

32 Weinshenker BG, Bass B, Rice GPA, et al. The natural history of multiple sclerosis: a geographically based study. 1 . Clinical course and disability. Brain 1989;112:133-46.

33 Ormerod IEC, McDonald WI. Multiple sclerosis presenting with progressive visual failure. $\mathcal{F}$ Neurol Neurosurg Psychiatry 1984;47:943-6.

34 Comi G, Filippi M, Martinelli V, et al. Brain MRI correlates of cognitive impairment in primary and secondary progressive multiple sclerosis. F Neurol Sci 1995;132:222-7.

35 Vleugels L, Pfennings LEMA, Pouwer F, et al. Psychological functioning in primary progressive versus secondary progressive multiple sclerosis. Br F Med Psychol 1998 (in press).

36 Thompson AJ, Polman $\mathrm{CH}$, Miller DH, et al. Primary progressive multiple sclerosis. Brain 1997;120:1085-96. 